\title{
RELATIVITY AND ITS PRECURSORS
}

\author{
BY ROBERT P. RICHARDSON
}

THE theory of relativity takes its root in a view which, though 1 a commonplace to all thoughtful modern scientists, accepted alike by Relativists and anti-Relativists, appears to the man in the street as a startling paradox. Even to the philosopher, if he belongs to a certain school of thonght, it has the appearance of a metaphysical heresy and one which he is liable to label by the title of relativism, a practice tending to confuse the issue when the relativity of modern physics is under discussion.

The view in question has to do with motion, something which in the opinion of the unscientific man is a self-given reality, like redness or sweetness, so that he can see no necessity whatever for inquiry into its nature. And there might indeed be no need of this if mankind had no more to do with motion than to gaze lazily at the movements taking place under our eyes. But such is not the case: science must measure motion with precision and be able to describe its course and its speed. That this may be done it is requisite for motion to be referred to something taken as standard of immobility, or, more precisely put, referred to a system of coordinates based on three non-collinear points taken as fixed and immovable. And the doctrine mentioned above, and which, as has been said, is not that which distinguishes relativity from other theories of physics, is that unless this prerequisite has been fulfilled it is nonsense to speak of motion in a scientific discussion: that motion is necessarily always motion relative to something.

In a somewhat broader sense, in which it is applicable to everyday life as well as to the domain of science, the doctrine is that motion cannot be intelligibly spoken of save as a change of observable phenomena, and indeed one consisting of change in distance, in direction, or in both, of something as regards something else. The view that motion has as its essence a change of observable phe- 
nomena was plainly set forth by I,eilmiz who, replying to the contention that "the fact of movement is independent of observation," remarked: "Mlovement is indeed independent of observation but it is not independent of olservability. There is no movement when there is no observable change. And in fact when there is no observable change there is no change at all." Leibniz contended that space was something purely relative, merely "the order of bodies among themselves." and regarded as absurd the doctrine of absolute space and the "fiction of a material linite tniverse promenading as a whole through an intinite enpty space." Leibniz, in short, took suldstantially the ground delineated by Sir John Herschell in the words: "Space in its nltmate analysis is nothing lut an assemblage of distances and directions." Ender this, the only rational view. the situation of a body in space is constituted by its distances and directions from other hodies, and it would be well to distinguish the position of a body from its situation. Just as a man is said to be in different situations when he is facing and when he has his back inrned to the olsserver (such a difference in posture having been regarded by Aristotle as of sufficient importance for consideration muncler a separate category) so a rotating sphere may be considered as co nstantly changing its position while remaining all the while in its original situation. The situation of a point of a body. usually called a "point of space" may for greater clearness be termed a site. $\therefore$ true point is the intersection of lines on a material body, while a trie line is the intersection nr boundary of surfaces and must be distinguished from the path through space of a moving point, often improperly called a "line," a distinction the importance of which will be manifest later.

The influence exercised by Leilmiz on the philosophy of physical science was far less than that of his great contemporary and rival. Newton, and the athority of the latter lent support to quite a different doctrinc. Newton refrained, he said, from defining time, space, place and motion because they were well known to everyone. Ile ackled, however, "Only I must olserve that the vulgar conceive these quantities under no other notions but from the relation the " bear to sensible objects." Newton held that, on the contrary, "In philosoplical disquisitions we onght to abstract from our senses, and consider things themselves, distinct from what are only sensible measures of them." He distinguished between relative 
and absolute place and motion, stating that "Absolute motion is the translation of a body from one absolute place into another; and relative motion the translation from one relative place into another." "Place is a part of space which a body takes up." "But because the parts of space cannot be seen or distinguished from one another by our senses, therefore in their stead we use sensible measures of then. For from the situations and distances of things from any body considered as immovable, we define all places: and then with respect to such places, we estimate all motions, considering bodies as transferred from some of these places into others. And so instead of absolute places and motions we use relative ones."

Measurability was, in point of fact, the chief characteristic of the new physical science that was being built up in the days of Newton. The new physics substituted precise quantitative conceptions of motion, mass, force, etc. for the vague qualitative conceptions of the Aristotelian and Scholastic theories. Relative motion, since it alone could be measured, was the only kind of motion really relevant in physical inquiries. A relative place can be determined by measuring its distance and direction from a given body : a relative motion likewise can be perceived and measured. But the alleged absolute place and absolute motion, those vestiges of the old physics which Newton strove to retain in the new, elude not only all measurement but even all perception. There is no way of specifying the absolute place of anything and none of measuring absolute motion: these cannot even be perceived. Indeed Newton himself remarked that "it may be that there is no body really at rest to which the places and motions of others may be referred." "No other places," said he, "are immovable but those that from infinity to infinity do retain the same given situations one to another: and upon this account must ever remain unmoved; and do hereby constitute what I call immovable space."

Newton does not appear to have considered the question of what absolute motion could be in itself. He took the naive stand that there was no necessity of inquiring into the nature of the absolute motion whose existence he affirmed, and apparently failed to see that in every case of motion there must be implied some sensible standard of reference, tacitly if not explicitly. Had he been able to cast off completely the trammels of the ancient teachings he would have realized that even when the adjective "absolute" is 
affixed, it is impossible to say intelligibly that a body is at rest or in motion fer sc. Such a statement has no meaning unless another plirase is adcled to the effect that the motion or rest is in reference to something else specified therein. Newton apprehended that the observable phenomena of recession or tendency of recession from a certain axis in a body (c.g. the earth) ought to be interpreted as evidence of that borly being in absolute motion of rotation around that axis, and so formulated the laws of mechanics as to necessitate this interpretation being made. Had he boldly taken these centrifugal plenomena not as eividences of motion in reference to nothing in particular lut as hasis for the very definition of "absolute motion of rotation" his standpoint would have been massailable. Plainly however he would thereby have been giving a new and decidedly nonnatural meaning to the word "motion," and this he was not prepared to do.

Following the lead of Newton, physicists for several centuries continued to speak vaguely of an "absolute motion" which was unmeasurable, while dealing solely with relative motion amenable to their measurements. Clarification of the topic did not come until the last half of the nineteenth century, and was due to two very remarkable men: Carl Neumann and Ernst Mach. These scientists, on consiclering the classical laws of dynamics which speak of uniform motion in a straight path, put the question whether or not these laws cotild be affirmed without reservation as to the reference system in view, and found the answer to be in the negative. And this is indeed quite obvious, for a path that is straight under some systems of coordinates will not be straight under others. Thus a body moving in a rectilinear path under a system of coordinates based on the sun as stanclard of immobility will not usually move rectilinearly when the earth is taken as standard of immobility. As an illustration of this suppose there to be a balloon suspended above the earth and rotating around a vertical axis. And suppose a body to move upward vertically from the earth to the balloon collinearly to this axis, striking the center of the bottom of the balloon while another hody also moving up vertically strikes the periphery of the bottom. Moreover suppose a third body to move horizontally towards the balloon in what is a straight path from the standpoint of earth as reference body, and let the three paths from this stand- 
point be of equal length. ${ }^{1}$ We have then rectilinear motion of three bodies over equal distances. All this is from the standpoint of a system of coordinates based on the earth, that is, based on taking the earth as standard of immobility. But suppose now that a new system of coordinates be adopted, based on the balloon as standard of immobility, this being regarded as stationary while the earth is supposed to rotate not around its ordinary polar axis but around the vertical axis passing through the center of the bottom of the balloon. Under this system of coordinates the first body will as before have moved in a straight path, but the second will have moved in a helix and the third in a spiral and these three paths will not be equal in length. And if the balloon, besides rotating as regards the earth, is moving upward vertically with a speed equal to that of the second body, then when change is made to the balloon as reference basis the path of the second body will be a closed circle. To visualize these facts we may suppose the second and third bodies to carry bits of lead pencils which trace the paths they have taken as marks on sheets of paper. The earth reference system will be represented by a vertical paper cylinder and by a horizontal sheet of paper, both attached to the earth. The balloon reference system will likewise be represcnted by a paper cylinder and a flat sheet of paper, but these will be attached to the balloon. Even a person unversed in the technique of analytical geometry can see in this way the difference between the paths under the two systems of reference and perceive that what will be straight under one coordinate system may be helicoidal or circular or spiral under another. And thus the impropriety of calling the path of a moving point a "line" is apparent. For a line is something absolute and immutable, entirely independent of the reference system in view, while a path is relative its character depending on what is taken as standard of immobility."

It is hence obvious that Galileo's law which asserts that a material point set in motion and then completely left to itself, no ex-

1Strictly speaking we should consider not the path of each body as a whole, but the path of some particular point on each body.

-Further discussion of this very important distinction between paths and lines and also a more thorough investigation of relativity, special and generai, will be found in the forthcoming second part of a work by the present writer in collaboration with Professor Landis: Fundanental Conceptions of Modern Mathematics, the first part of which, I ariables and Quantities, was published by the Open Court Publishing Co. some years ago. 
ternal influences acting tpon it, will move in a straight path and pass over, in equial times, equal distances measured along the path, is incomplete and indefinite. For it does not say what reference system is in view. The law assuredly cannot hold under all reference systems, for if a given material point is, in conformity with the law, moving uniformly in a straight path from the point of vicw of one reference system, we can always find another reference system under which it is not moving in a straight path, so that under this latter system the law is heing violaterl. Here many scientists were inclined to imagine they could cut the Gordian knot and brush the whole problem asicle with the remark that of course the laws of dymamics are to be construed as referring to the true standard of immobility. But the question at once arises: What is the true standard and why is it to be accepted as true? In what sense, for instance, can it be asserted that taking the sun or the "fixed" stars as standard of immobility is any "truer" than taking the earth as standard? In what sense is it legitimate to say that it is "true" that the earth revolves around the sun and rotates on its axis and "false" that the earth is stationary and the sun and fixed stars revolve around it in immense orbits once every twenty four hours? Motion of bodies is essentially change of distances or directions between them. And if we have in view two bodies with which such change takes place we can efually well say that the first is moving and the second stationary, that the first is stationary and the sccond moving, or that both are moving. The observed facts, the changes in distance and direction, remain the same: we are merely using three different ways of describing the same set of facts.

The problem then is far more sulthe than the casual thinker might imagine, and it was solved in one way by Neumann and in (quite another by Mach. Nemmann held fast to the classical theory of mechanics and contended we must, to justify this, postulate the existence in some tnknown part of space of a body unknown to us (called by him Npha) which is absolutely rigid, its shape and dimensions being unchanged through all eternity. He conceded indeed the possibility of basing an "Alpha" reference system not on a perfectly rigid body but upon the "three so-called axes of inertia of " material body" whose shape and dimensions were stuject to change, and even the possibility of the system Alpha being constituted by the chief axes of inertia of the universe, but neither 
the sun nor any known "fixed" star can be taken as standard of immobility; none of these can be regarded as stationary if the classical laws of mechanics are to hold good. A body then is to be deemed "truly" at rest or "truly" in motion according to whether it is at rest or in motion as regards Alpha. The Alpha system of coordinates is the standard of "absolute" motion, and the laws of mechanics must be construed with this reference system in view. Neumann considered also the standard of time. Since time is measured by motion. the classical statement that a moving material point, influenced only by its own inertia, will pass over equal distances in equal times presupposes a given motion taken as standard-as the type of uniformity. And to obviate this difficulty Neumann proposed to modify the statement in question by making it assert that two moving material points, each of which is left to itself, will move in such a manner that every distance travelled by the one always corresponds to (i.e. is isochronous with) an equal distance travelled by the other.

Neumann's procedure is evidently tantamount to taking the classical laws of mechanics as constituting an implicit definition of absolute motion. We must, he holds, take as the "true" system of reference one which makes these laws hold good. Mach however-looked at the matter from a different angle, and took the standpoint of relativity, rejecting absolute motion altogether. "Obviously," said he, "it does not matter whether we think of the earth as turning round on its axis or as at rest while the celestial bodies revolve around it. Geometrically these are exactly the same case of a relative rotation of the earth and of the celestial bodies with respect to one another. Only the first representation is astronomically more convenient and simpler. But if we think of the earth at rest and the other celestial bodies revolving round it, there is no flattening of the earth, no Focault's experiment, and so on-at least according to our usual conception of the law of inertia." The difficulties which Mach found in the law of inertia, he remarks, "exactly coincide with those of Neumann" but the latter's solution was not the only one possible, there being really two alternatives: "Either all motion is absolute or our law of inertia is wrongly expressed. Neumann preferred the first supposition, I the second." The law of inertia, Mach contended, ought to be so conceived that 
exactly the same thing results from the supposition of the earth as stationary as from the usual supposition that it is moving.

The thesis thus set forth by Mach is the standpoint of relatizity, of which what is now known as the theory of relativity is merely the development in a perverted way. Thus the most important feature of the general relativity theory, its so-called law of gravitation, which is not really a law of gravitation at all but a law of motion: is a realization (though an imperfect one) of the expectations of Mach that "integral laws, to use an expression of C. Neumann, wili some day take the place of the laws of mathematical elements, or differential laws, that now make up the science of mechanics, and that we shall have direct knowledge of the dependence on one another of the sitrations of brdies. In such an event, the concept of force will have become superfuors."

The constant coupling of the word "relativity" with the name of Dr. Albert Einstein has made prevalent the erroneous impression that relativity originated with that phycicist, and if the casual reader were asked to whom the theory of relativity was due he would be likely to accredit the alpha and omega of it to Einstein. This however is a gross error. The standpoint of relativity, as we have seen, originated with Ernst Mach, and even the speculations of that particular school of thought of which Einstein is the most prominent figure cannot be accredited to him alone. Thus, with what is known as the special theory, if we consider as paramount factor not the detail work but the guiding thoughts by which this was inspired, then the father of this special relativity theory was undoubtedly Henri Poincaré.

Einstein, in working out the details of his special theory. followed precisely the path previously mapped out by Poincaré as suitable for developing a theory on the basis of relativity. It was Poincare who pointed out the importance in such a scheme of the mathematical formulas of the Dutch physicist. H. A. Lorentz (who had incleed been anticipated by W. Voigt in 1887) and introduced the name "Lorentz transformation," so familiar to the reaclers of Einstein's works. These formulas were at Einstein's disposal, though it is claimed he had not read Lorentz's work, published in

3The fundamental equation of Einstein's theory of gravitation does, it is true, involve ten coefficients sometimes said to represent the potentials of gracilation, and the subsidiary equations by which these coefficients are determined are called the field equations of grazitution, but in these phrases the word "gravitation" does not have its classical sense. 
1904 , before writing his own fundamental article. This fundamental article by Einstein. Zur Elektrodynamik bezegter Koerper, did not appear until 1905, but as early as 1898 Poincaré (in La Mesure du Temps) had given utterance to the primary thought of the special theory of relativity: that we cannot intelligibly speak of the simultaneity of two events occurring at places far apart without some convention, constituting a criterion of simultaneity, and equivalent to signalling between the two places, a signalling which would most naturally be conceived to be carried on by means of light rays. This, Poincaré noted, led to the doctrine that "The simultaneity of two events, or the order of their succession, the equality of two durations, are to be so defined that the enunciation of the laws of nature may be as simple as possible." He also pointed out the possibility of basing a "new rule for the investigation of simultaneity" upon the postulate that "light has a constant speed, and in particular that its speed is the same in all directions." This postulate which, somewhat enlarged, is one of the fundamental assumptions of special relativity, Poincaré asserted (though erroneously) was one without which no measurement of the speed of light could be made. It "could never be verified directly by measurement : it might however be contradicted by this if the results of different measurements were not concordant."

Poincaré (in La Theorie de Lorenta et le Principe de la Reaction) envisaged correlating physical phenomena no longer to true time but to Lorentz's local times, and put forward the doctrine that the laws of nature ought in their mathematical formulation be covariant as regards Lorentz transformations. He foresaw the rise of a new mechanics in which "the inertia increasing with the speed, the speed of light would be a limit beyond which it would be impossible to go" (The Present and Future of Mathematical Physics, a paper read before the Congress of Arts and Sciences at St. Louis in 1904.) It was Poincaré who first made use of the name "postulate of relativity," which, however, he defined as the law of the impossibility of finding experimental evidence for the absolute motion of the earth. The theories of Minkowski, Einstein's most important coworker, likewise constituted a following out of Poincare's ideas. In 1905, three years before Minkowski took the field, Poincaré, in L'espace et la Temps, remarked that it might be advisable to abandon the old view that time and space were "two entities entirely 
distinct which can be imagined separately" and to regard them as "two parts of the same whole and two parts which are so closely" interworen that we conld not easily separate them." And he voiced the thought that in the representation of motion the time concerned miglit appear as a fourth and imaginary coordinate of space. Even the relativist dogma that gravitation cannot be propagated instantaneously was foreseen by Poincare who pointed out that "in this new mechanics there is no effect which is transmitted instantaneously."

In the general theory of relativity the basic thought is that of Mach, aiz. the replacement in dymanics of the law of gravitation by a law of motion. But in what Einstein built upon this basis the influence of Poincaré is again manifest. Einstein, in consirlering the motion of hodies in gravitational fields, where they do not move uniformly over rectilinear paths, brought into play the conception of a local puckering of "space-time," a deviation from its normal character, to an extent depending on the strength of the gravitational field, as an interpretation of this deviation from the normal motion of a body, that is, its motion where no gravitational influence is apparent. And this is an obvious ontgrowth from Poincaré's doctrine that if there were observed anomalous phenomena in the transmission of light from distant stars which could be described as deviation of light rays from their normal rectilinear courses. this could also be interpreted as a non-Eucliclean structure of (a non-zero curvature of ) space. And in view of all these facts one does not know at which to be the most astounded: the magnanimity of Poincare who was always over-anxious that there should be recognition of the labors of those who hacl reaped where he himself had sown, the apathy of his friends after his cleath, or the peculiar attitude of Einstein and his coterie, exemplified by Born oi Goettingen, who refers to Poincare as one of those who "collaborated" with Einstein in the development of the relativity theory!

For years Mach battled for the standpoint of relativity almost single-handed and under great discouragement. When he brought to the attention of physicists the "indefiniteness, the difficulties and the paradoxes" found alike by Nemmann and himself in the law of inertia in its classical form he encountered, he says, "dislain and surprise" from almost all the physicists with whom he discussed the subject. This is in striking contrast with the enthusiastic re- 
ception given the "relativity theory" of to-day. The contentions of Nach and Neumann, having on their side merely irrefutable logic were disdained. Relativity theory, so-called, notwithstanding the fantastic and illogical features it embodies, has been acclaimed becanse of the allegation that certain predictions made by it, certain consequences flowing from it, have been verified by astronomical observations. Unfortunately in the eyes of many "scientific" men, such a verification of the consequences of a theory is sufficient to prove the truth of the premises from which these consequences follow, no matter how preposterous these premises may be in themselves. This psendo-scientific mentality is undoubtedly due to the divorce of science-teaching from logic. It is no exaggeration to say that ninety per cent of college graduates in science are unaware that true conclusions can follow from false premises by perfectly sound processes of reasoning, and that at least fifty per cent of the professional teachers of science are not alive to this elementary logical truth and to its bearing on scientific hypotheses.

Mach's attitude towards "the Relativists" was an unfavorable one. In his Principles of Physical Optics he said: "I must....as assuredly disclaim to be a forerumner of the relativists as I withhald from the atomistic belief of the present day." And le promised to set forth in a later work "the reason why and the extent to which, I discredit the present-dav relativity theory, which I find to be growing more and more dogmatic, together with the particular reasons which have led me to stch a view-the considerations based on the physiology of the senses, the theoretical ideas, and above all the conceptions resulting from my experiments." This repregnance to stand as godfather to the divagations of the Relativists is natural, but it must not blind us to the fact that the "relativity theory" is unquestionably an offshoot of the doctrines tanght by Mach. The quintessence of Mach's standpoint of relativity is embodied in his protest against the classical formulation of the law of inertia becarse it failed to take into account systems of coordinates based on reference bodies rotating (or in other difform motion) as regards the Alpha system, but could be asserted only when the system of reference in view was Galileian, that is, was based on Alpha or on some other system of coordinates which, as regards Alpha, was either at rest or in uniform motion of transla- 
tion. And we can only regard as a development of this the more sweeping demand put forward as the General Principle of Relativity, the postulate that "The laws of physics must be so constituted that they remain valid for any system of coordinates moving in any manner."

The theory of relativity, as set forth by Einstein and his school, is subdivided into two parts. The first, the theory of special relativity. deals solely with Calileian reference systems, and in it there is enunciated the Special Postulate of Relativity which runs: "If a system of coordinates. $K$, is chosen so that in relation to it physical laws hold good in their simplest form, the same laws also hold good in relation to any other system of coordinates, $\mathrm{K}^{*}$, moving in uniform translation relatively to $\mathrm{K}$." Thus in the special theory of relativity there are privileged reference systems which are alone consiclered. And in the use made of this postulate it is assumed. rather gratuitously, that $w$ hen $K$ is chosen in accordance with these specifications it (and hence all the other privileged reference systems) will be Galileian. That is, precisely the same reference systems are griven special privileges in the Special Theory of Einstein as in the Galileian-Newtonian Theory. By the side of his special relativity postulate Einstein puts forth a postulate concerning the speed of light to the effect that, when in a vacuum and uninfluenced by gravitation, every ray of light moves, as regards what he calls "the stationary coordinate system" (i. e. whicherer Galileian coordinate system happens to be first taken into account) in a rectilinear path with the constant speed of $c$ (that is about 186000) miles per second, regardless of whether the light is emitted from a body at rest or from one in motion. And here, of course, he is deviating from the classical theory of light propagation, for by this the speed of light was not understood to be 186000 miles per second under whichever Galileian coordinate system we might choose to start with: the speed in the classical theory would be accepted as 186000 miles per second only as regards a reference system which makes the hypothetical light-transmitting medium stationary. Nor is there in the classical theory of optics anything which identifies this particular reference system with the suppositious system as regards which the heavenly bodies have the "absolute motion" imagined by Newton. It is a misrepresentation of fact to say, as Einsteinians sometimes do, that in pre-Relativist days the luminiferous 
ether was taken in classical physics as the basis for an absolute system of coordinates; the thought did not even arise of calling in optics to aid the theory of mechanics.

The Relativists give their special postulate an interpretation. where light is concerned, which makes this innocent looking proposition one pregnant with paradoxes. For they apprehend that if "the same laws" are to hold good with all the different Galileian reference systems then the speed of light must always be $c$ miles per second, no matter which Galileian reference system be under consideration. That is, if a ray of light is moving out from a Galileian reference bedy (i. $\mathcal{c}$. a body which when taken as standard of immobility gives a Galileian system of coordinates) with a speed of 186000 miles per second towards a body which moves rectilinearly to meet it with a uniform speed of 93000 miles per second. then, according to the Relativist view, when this second body is taken as a new standard of immobility (giving rise to another Cralileian system of coordinates) the speed of the ray of light measured with reference to the new system cannot be 279000 miles per second as pre-Einsteinian thinkers would have opined, but must still be merely 186000 miles per second. Likewise, no matter how fast a body may be retreating from the source of light, the speed with- which a ray of light overtakes it must, whether measured with reference to the source or with reference to the receding body, be always 186000 miles per second. Again, if two bodies, each so moving that it is capable of being taken as a Galileian reference body, clash at a point as they pass one another, emitting then a single wave of light, this wave, according to both the classical and the Einsteinian theory, would spread out in the form of a luminous spherical shell. In the classical theory the center of this shell would be either the point of the first body which clashed with a point on the other, or the point of clash on the second body, or neither, according as either the first body or the second body or neither was stationary as regards the ether. But according to Relativist doctrine, if the first body be taken as standard of immobility the point on it is and remains the center of the spherical shell, while if the second body be taken as standard the point on this body is and remains the center, and this will continue to be the case even after the two bodies have moved a few million miles apart. Since it is by a mere fiat of the human mind that either body is made standard of immobility, 
this doctrine will seem to the laynan very much like the assertion that a sphere has two centers. It can, in iact, only be maintained by special Finsteinian methods of measuring distances. And such manipularion of measurement. which is applied not only to distances of space but also to lengths on bodie's and to durations of time is an essential ieature of relativity theory. That it is possible to lay down conventions of measurement under which distances. lengths and durations are measured in various eccentric ways must be conceded. For instance there is no logical inconsistency in some one laving down the fiat that the measurement of all lengths and distances is to be based on a rubber yard-stick which he holds in his hand and takes as standard of length. He can then assert that as he stretches his yard-stick Paris and London come closer and closer together. and that he can mate the number of miles between them to he on!y half what it was before. This statement, properly understood. is undeniable, but it is not a fact of any moment, and the systen of measurement under which it is true is in no way useful to mankind. The rubber yard-stick of the Relativists is their doctrine that light always has the speed of 186000 miles per second as regards every Galileian reference stem whatsoever. This they set up as a veritable fetish. demanding that science so adjust all its measurements as to make this dogma hold good. Here at once it becomes evident how far a rational standpoint of relatiaty is irom affording support to the special postulate of relativity. According to the former we ought indeed be able to describe the phenomena of nature from the point of view of any reference system we choose to adopt, and taking any particular reference system it ought to be theoretically possible to formulate the laws of physics with this as basis. But it would he taking an umjutifialsle step in the dark to pass from this to the Finsteinian dectrine that the general laws of physics can and ought to he so formulated as to be "the same" when different reference systems are in culstion, and to regard this sameness as repuiring light to have the speed of 186000 miles per second no matter what ralileian system of coordinates be in riew. And still more serious is the step taken in the general theory of relativity where sameness of general laws is (again on a priori grounds) required alite for Galileian and non-Galileian reference systems. the "sameness" stipulated here being technically described as "covariance of the general equations of physics towards all trans- 
formations of coordinates." Relativists argue that a particular coordinate system is merely a particular way of observing nature, and that as it is unthinkable that an olserver can change the crunse of nature by merely changing the point of view from which he looks at it, the general laws of nature must be "the same" 11nder all systems of coordinates. Yet, strange to say, they hold that the length of a hodly and the duration of an event can be and is changed by merely looking at it in a different way-by a mere change in the coorlinate system under which it is viewerl-this contention, which would overthrow completely the mensuration of classical science, being necessary to "1phold the Relativist riew under the interpretation Relativists give to the phrase "sameness of law."

The scientific methols of measurement which the Relativists ask us to abandon rest essentially on the assumption that devices for measuring lengths, durations. ctc. are not affecterl by mere displacement in time or space or by mere change of the reference system in view. The affirmation that the length of a measuring rorl, and in general the size and shape of a body, is not changerl by transporting it to a rlifferent situation is termerl the . Ixiom of Free Nobility, and is one of the basic principles of geometry as that science has hitherto been understoorl. One kind of change would indeed be of indifference and that is a change of all hodies, measuring rods includerl, in the same proportion. For then the results of measurement would be invariable, and such a change would be mperceivalile by the senses and coukl not be noterl. In fact it cannot be enrisaged even as a possibility if we take the somnd philosophical gronul that perceivability is the sine gua non of fact. The Axiom of Free Molility then merely asserts comsistency of measurements, of comparisons marle at different places, and is sometimes more properly set forth as the Axiom of Congruence, asserting that if two geometrical figures are congruent (can be made to fit exactly on one another) at one place and time they will also be congruent at every other place and every other time. Tikewise in measuring durations there is assumed an Axiom of Consistency in Synchroneity to the effect that regular natural processes which are synchronous at one part of space and at nne time will be synchronous at every other: that, for instance, a chemical process which measurerl by a clock takes $t$ scconds at one place and time will at another place or another time or at another place and another time again refuire 
precisely $t$ seconds for its completion on timing it by the same clock. Science does not, it is true, assume that there are any wholly rigid and invariable bodies making perfect measuring rods or any perfectly rumning clocks. liut it does assume that variations in the standard of measurement can be compensated and allowed for. and that these variations are never die to mere space or time or to the reference system adopted but are caused by other observalble phenomena such as impressed force, temperature, etc.

A much deeper and more coherent thinker than Einstein (and one of his critics) Paul Painlerc, has pointed out that all science has hitherto been based upon what he terms the P'rinciple of Causality, namely "When the same conditions are realized, at two different instants, in two different places of space, the same phenomena ahways reproduce themselves displaced solcly in space and time." 4 This principle presupposes the existence of methods for measuring lengths, durations, etc. and is only true when suitable conventions of measurement have been adopted. And thus the principle has as an implication that "It is possible to adopt once for all and for all phenomena a measure of length and a measure of time such that the principle of cansality will be true always and everywhere." To adhere to classical geometry is to measure lengths etc. in a manner suitable to the needs of the science, and the acceptance of classical mechanics implies the assertion that "It is possible to adopt once for all and for all the motions of the universe a method of reference such that the Axioms of Mechanics will be true always and everywhere," this being substantially the view of Neumann. The difference between the classical point of riew and that of relativity may be well strmmed up by putting what we shall call the Postulate of Painler'e in antithesis to the Postulates of Einstcin. Luder the former name we put forth this assertion: The conzentions of measurement adopted for the primary quantitics of physics, aiz. lengths, durations, ete. ought to be so framed that these attributes preserie constancy in z'alue when there is a change in place, a change in time. or a change from one coordinate sy'stem to another. This roices among other things our determination to adhere to the law of tree mobility. It is not, of course, sufficient for insuring consistency of

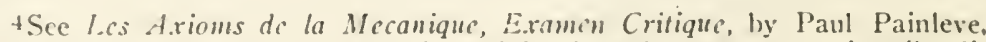
1922. in particular PP. 9, 11 and 23. Painleve's strictures were primarily directed against the general theory of relativity, but apply with equal force to the contractions of lengths and dilations of disations of the special theory. 
measurement of distances along paths of motion of points in space (as contradistinguished from lengths of lines on bodies), and if this be desired it is necessary to add a clause recognizing certain particular reference systems as alone admissable in physical science. Science must choose then between the Postulate of Painleve and the Postulates of Einstein: the latter is nint compatible with the former. and sacrifices consistency in measurement to covariance in equations.

Relativists, of course, take the ground that their postulates and the conventions of measurement required to justify them are not the result of an arbitrary fiat but flow naturally and inevitably from the observation of nature. And it is hardly necessary to say that they are partictlarly prone to make use of the Michelson-Morley experiment as an argument. Dr. Carus, commenting on this, remarked: "What this famous experiment has to do with the principle of relativity except in a most general way is not yet clear to those who have not joined the ranks of the relativity physicists: but the relativity physicists insist very vigorously and dogmatically that it proves or at least favors their theory. "J The Relativists bring the Michelson-Morley experiment into play as upholding their special postulate, but in point of fact the failure to detect an "ether drift" by this experiment has no logical connection whatever with the special relativity theory. For the experiment was carried ont on the earth, and the earth as reference body is not Galileian, and thus has no concern with the special theory. If indeed it could be shown that an ether drift was never to be found with a certain Galile:an reference body this would have a bearing on the doctrine that the speed of light is invariably 186,000 miles per second, no matter what Galileian coordinate system it be measured under. But to base this conclusion on the behavior of a non-Galileian reference body is what is known in logic as an ignoratio elcnchi. This being the case we need not stress the fact that transmission of light on the earth's surface is not through a vacuum and does not take place in the absence of gravitation. Nor need we point out that the failure to observe an anticipated ether drift in an experiment can be given various interpretations. Indeed Dr. L. Silberstein remarked that what we have learned from the Michelson-Morley experiment "stripped from every theoretical interpretation" is merely p. 68.

5The Principle of Relativity, Chicago, The Open Court Pub. Co., 1913. 
as follows: "Let O, A, B, be three points marked on a slab of stone. Then whatever the phase-difference or time-lag between the light signalling OAAO and OBBO in one orientation of the slab, it remains the same under any other orientation say, after turning the slab by $90^{5}$ relatively to the Earth, that is, and no matter at what season of the year."

The basic feature of Relativist mensuration is its thcory of simultaneity And the primary idea here is that simultaneity, not merely as an occasional necessity of practice, but as a matter of principle, must, with events not in the same neighborhood, be determined by means of light signals flashed from the places where the events take place-that the very definition of simultaneity in such cases rests on the laws of the transmission of light. Relativists thus repudiate with disclain the doctrine of classical science that simultaneity of occurrences at different places is to be defined by means of clocks which, having run synchrononsly when together, may he taken as still synchronous when widely separated. This path to the definition of simultancity by way of the doctrine of the constant speed of light was first pointed out by Poincaré who remarked that the postulate "that light has a constant speed and in particular that its speed is the same in all directions" could be taken as founclation for the notion of simultaneity. Acceptance of the postulate he based on the Principle of Sufficient Reason, that is to say, becanse there is no sufficient reason why any particular direction should possess particular advantages for the propagation of light, we may legitimately hold that all directions are alike in this respect, and that in all of them light has the like speed. This postulate he asserted was one without which no measurement of the speed of light could be even attempted. Yet in point of fact it is (puite () l)vious that measurement of the speed of light could readily he made under the assumption that the speed of light was not constant provided some definite law was laid down fixing the relation of its speed in one direction (or under one set of circumstances) to its speed in every other. And if certain observations could be interpreted either as a variation in the speed of light or a varia(if'hil. Wag., 1924, 48, p. 397. Noreover Miller (Science, 1926, 63, p. 434) has pointed out that the first nonchalant assumption of the Relativists that the Michelson-.Morley experiment gave a true zero or null result, showing no difference in the light signalling under different orientations of the apparatus, is very far from the truth. The experiment has never yielded a really nuli result. The fact is simply that such an "etiser drift" as might be anticipated under the assumption that the ether awas stationary as regards the sun or the fired sturs did not make itself manifest. 
tion of lengths and of durations under the influence of mere change in time, in place or in reference systen, then a physicist who adhered to the Postulate of Painleve would accept the former alternative.

Relativists however take the reverse point of view and begin by considering a Galileian reference system in which the "postulate of the constant velocity of light" is understood to holkl. A body moving longitudinally in uniform translation with respect to this gives the basis for a second Galileian reference system. The latter is nsually likened to a train, which when itself taken as standard of immobility is stationary, while the reference body of the other system is not mnlike an embanknent alongside which runs the "train" which is, of conrse, moving as regards this system of coordinates. At the instant the front of the train is opposite a point $\triangle$ on the embonkment a flash of light is sent thence rearward, and at the instant the rear is opposite a point $\mathrm{B}$ a flash is sent from here forward. And the question is posed whether or not the passage of A by the front and that of $B$ by the rear of the train are simultaneous events. This question is considered as equivalent to that as to whether the two flashes do or do not simultaneonsly reach an observer midway between their origins. Now there might be two snch observers, one in the middle of the train, the other on an emlankment, halfway between $A$ and $B$. If then the light signals reach the embankment-observer sinultaneously it follows that they could not reach the train-observer simultaneously, for he is moving to meet the flash emitted from the front while moving away from the flash which is striving to overtake him from the rear, hence the latter will reach him later than the former. This, to be sure, is merely an argmment ad homincm, for it is based on the classical view that the motion of an observer towards or away from a source of light makes a difference in the time it takes this light to reach him, a view repudiated by the Relativists themselves who regard each observer as making use of a reference system under which he himself is stationary and under which light coning to him has always, as regards this system, the constant speed of $c$ miles per second. The argument takes the ground that as regards the embankment-observer the light flashes have e.r hypothesi this speed ( since it has been stipulated that the "postulate of the constant velocity of light" holds as regards the embankment coordinate system) but do not have it as regards the train-observer. But under 
the special theory of relativity the light flashes would have the selfsame speed for both observers, and ought to reach both simultaneously. The Relativists contend that they have here demonstrated the relativity of simultaneity and have shown that we should never speak of two events as simultaneous in an absolute sense but only as simultaneous when a given reference system is in view. But in truth the conclusion thus drawn is based on premises taken from two conflicting theories: the Relativist doctrine that simultaneity must be defined by means of light signals, and the classica! doctrine regarding the effect produced by motion of an observer towards or away from a source of light. None the less the Rclativists with arguments such as that just outlined, deem that they have overthrown the time-honored theory of chronometry, and justified their demands for the acceptance of the two postulates of the special theory. From the doctrine of relativity of simultaneity they proceed to that of relativity of lengths and durations, and contend that when change is made from a Galileian reference systen in which a rod is stationary to one in which it is moving longitudinally, its length contracts and that there is an analogons dilation of durations, the duration of any even taking place on one Galileian reference body and measured in the first instance as regards this, measuring up as a longer interval of time when considered from the point of view of another Galileian reference body moving relatively to the first. In the case of the rod, the ordinary method of measuring by direct application of a measuring stick is accepted as satisfactory in the first reference system, but with the second it is contended that the measuring stick must be applied to something stationary as regards the new reference system. That is, two points are determined on the "embankment" of the second system which are simultaneously opposite the front and rear respectively of the rod. And the length measured between these two points is declared to be what we must take as the length of the rod, so that if simultaneity is relative, length may well be so deemed. This declaration however rests only on the ipse dirit of the Relativist. And science can-if it accepts the Postulate of Painleve. acill-continue to regard the length as, in principle at least, determined by direct measurement, notwithstanding the change to a new standard of immobility under which both the body measured and the measuring stick are alike in motion. 